\title{
Firmness of purpose in life significantly influences emotional state and autonomic nervous activity
}

\author{
Riichiro Ishida*, Tsueko Abe, Masahiko Okada
}

Division of Clinical Preventive Medicine, Niigata Graduate School of Medical and Dental Sciences, Niigata University, Niigata, Japan; ${ }^{*}$ Corresponding Author: ishida-riichiro@hb.tp1.jp

Received 23 April 2011; revised 2 June 2011; accepted 15 July 2011.

\begin{abstract}
The present study was performed to clarify the influence of having a firm purpose in life (PIL) on psychological and physical stresses. Participants were 59 subjects (19 males and 40 females) with a mean age of $\mathbf{3 0 . 9}$ years (standard deviation, 12.1; range, 18 - 68 years). We used a mental arithmetic (MA) test as a goal-oriented stressor; the test was supposed to be finished before a time limit expired without using pencil and paper. We used a PIL test to assess the intensity of personal meaning in one's life, an interest in mental arithmetic test (IMAT) to assess the intensity of interest in the MA test, and an anxiety inventory (AI) to assess the anxiety level of before and during the MA test. Heart rate (HR), systolic blood pressure (SBP), diastolic blood pressure (DBP) and nose tip temperature (NTT) were recorded before and after MA. We used logistic regression modeling to estimate the odds ratio (OR) of independent variables of age, sex, PIL test score, Al score, HR, SBP, DBP, and NTT before the MA test. Multivariate-adjusted models showed that PIL test scores prior to the MA test significantly predicted the observed changes in AI score and HR. Other variables were not significant predictors. In conclusion, we found that a firm PIL decreased anxiety under psychological stress as measured with the MA test, even with objective evaluation by others. Having a firm PIL also lowered the response of the sympathetic nervous system to psychological stress.
\end{abstract}

Keywords: Purpose in Life; Psychological Stress; Anxiety; Sympathetic Nervous Activity; Longevity

\section{INTRODUCTION}

Physical and psychological stresses can easily cause anxiety and simultaneously activate the sympathetic nervous system [1,2]. A "will" to seek meaning in life or to achieve purpose in life (PIL) is significantly associated with intrinsic motivation to get satisfaction and/or pleasure from an act $[3,4]$. Hence, persons with a firm PIL are less sensitive to physical and psychological stresses [5]. People exhibiting a Type A behavior pattern, characterized by traits such as impatience, aggressiveness, strong approval motivation, and a chronic struggle to achieve one's goal, are much more sensitive to stressors $[6,7]$. It has been reported that type A behavior tended to increase sympathetic nervous activity, generate reactive oxygen species, and cause secretion of $\beta$-endorphin $[1$, 6-8]. It is also known that type A behavior is associated with increased risk of coronary heart disease (CHD) [7].

Several tests have been devised to measure the intensity of PIL. Crumbaugh and Maholic, for example, developed a PIL test to assess the intensity of personal meaning in one's life [9]; Sato and Tanaka modified this test for Japanese people [10]. This test enables measurement of intrinsic motivation, satisfaction, or pleasure in life. Audio-visual stimulations such as watching a kaleidoscopic roller coaster movie have been used to show that persons with firm PIL exhibited less anxiety and had lower sympathetic nervous system responses compared with persons with less firm PIL [5]. The stimuli were physical and continuous without any appointed goal. In daily life, however, there are many goal-oriented stresses involving time limitations and being exposed to objective evaluation. For example, company employees have to accomplish a large number of tasks in a day, students have to complete their homework assignments, and housewives have to do many routine tasks every day.

The present study was performed to clarify the influence of having a firm PIL on responses to psychological and physical stresses. We hypothesized that people with a firm PIL show low anxiety and low sympathetic nervous response to psychological stress. 


\section{METHODS}

\subsection{Study Population}

Details of the subjects and the experimental protocol have been described previously [11]. Briefly, 62 subjects (20 males and 42 females) in a variety of occupations, including housewife, salesman, elementary school teacher, and technician, were recruited. Of these 62, 3 subjects whose attitude and physical symptoms could seriously affect the test results were excluded due to excessive talking during the test, frequent arrhythmia, or excessive time spent reading documents because of presbyopia. Finally, 59 subjects (19 males and 40 females) with a mean age of 30.9 years (standard deviation, 12.1; range, 18 - 68 years) participated in the study.

The subjects were instructed not to drink alcohol or tea and not to smoke on the day of the experiment. No instructions were given regarding food intake. The test was performed on an individual basis. The ethics committee of the Faculty of Medicine of Niigata University approved the study, and all subjects provided written informed consent.

\subsection{Goal-Oriented Stress Test}

The goal-oriented stress we applied was a mental arithmetic (MA) test that was supposed to be finished within a given time limit without using pencil and paper. An instructor (male, 57 years of age) administered each test. First, the instructor explained the written protocol on the panel as follows: "Please perform the mental calculations as quickly and accurately as possible in four minutes. Answer the resulting values when I say 'stop and answer.' Do not talk during the test other than to answer with the resulting values during the test." Then the instructor randomly chose one of three cards: "1000 - 6," "1000 - 7," or "1000 - 8," and showed the card to the subject. Subjects were requested to perform sequential subtractions as shown on the card. Thus, for example, a subject who received the " $1000-6$ " card would calculate $1000-6$ (=994), $994-6$ (=988), $988-6=982$, and so forth.

\subsection{Psychological Tests}

The PIL test used here consists of 20 items to which subjects expressed agreement or disagreement on a 7-point Likert scale. The questionnaire is outlined in Appendix $1[5,10]$. High scores indicate a firm PIL [10]. The subjects were divided into groups according to their scores: firm PIL (FPIL) ( $\geq 55$ points) and weak PIL (WPIL) ( $<55$ points).

We also devised another two tests. One was the interest in mental arithmetic test (IMAT) to assess the inten- sity of interest for the MA test. The IMAT consisted of only one question, "Are you interested in mental arithmetic?" and was scored on a 6-point Likert scale (1: not at all, 2: very weakly, 3: weakly, 4: somewhat strongly, 5: strongly, 6: very strongly). The other was an anxiety inventory (AI) to assess the anxiety level of before and during the MA test. The test before MA consisted of the question "Do you feel anxiety before MA?" and the test during MA was the question "Did you feel anxiety during MA?" These tests were each scored on a 6-point Likert scale (1: not at all, 2: very weakly, 3: weakly, 4: somewhat strongly, 5: strongly, 6: very strongly).

\subsection{Measuring Autonomic Nervous Function}

After the subject had sat in a chair comfortably for 20 minutes, heart rate (HR), systolic blood pressure (SBP), and diastolic blood pressure (DBP) were recorded using an electric sphygmomanometer (ES-P1000, Terumo, Tokyo, Japan) and nose tip temperature (NTT) was measured with a skin thermometer (D717, Technol7m Tokyo, Japan). These measurements were performed before and after the MA test. As environmental conditions affect the autonomic nervous function, room temperature, humidity, illumination and noise were controlled at $24.51{ }^{\circ} \mathrm{C} \pm 1.02^{\circ} \mathrm{C}, 58.64 \% \pm 4.61 \%, 259.17 \pm$ $78.21 \mathrm{~lx}$ and $53.10 \pm 1.37 \mathrm{~dB}$, respectively. Atmospheric pressure was measured at the time of the experiment.

\subsection{Statistical Analyses}

Univariate analyses were performed first to compare age, sex, PIL test score, IMAT score, and environmental conditions such as room temperature between FPIL and WPIL groups. Then AI score, HR, SBP, DBP and NTT were compared between values observed before and during/after MA tests.

A chi-square test was used for evaluating differences in the number of men and women between the FPIL and WPIL groups. An unpaired 2-tailed $t$ test was used to evaluate differences in age and environmental conditions such as room temperature between the FPIL and WPIL groups. The Mann-Whitney Test was used for evaluating differences in IMAT score between the FPIL and WPIL groups. The Wilcoxon signed rank test was used to evaluate changes in the AI score from before to during the MA test. Paired 2-tailed $t$ tests were used in evaluating changes of the four autonomic indicators HR, SBP, DBP, and NTT before and after the MA test. The basic characteristics were expressed as mean \pm standard deviation (SD).

Multivariate analyses were performed to evaluate changes before and during/after MA tests as follows. 

test)

Change in AI score $=($ during MA test $)-($ before MA (est)

Change in HR, SBP and DBP $=($ after MA test $) /$ (before MA test)

Change in NTT = (before MA test) / (after MA test).

We used logistic regression modeling to estimate the odds ratios (OR) of independent variables for age, sex, PIL test score, AI score, HR, SBP, DBP, and NTT before the MA test. Subjects with PIL test score $\geq 55$, AI score before $\mathrm{MA} \geq 2.05, \mathrm{HR} \geq 64.03, \mathrm{SBP} \geq 107.49, \mathrm{DBP} \geq$ 72.88 or NTT $\leq 33.85$ before MA were assigned a value of 1 for each respective independent variable; otherwise a value of 0 was assigned. For dependent variables, a value of 1 was assigned 1 for each subject whose score changes were: $\mathrm{AI} \geq 1.10, \mathrm{HR} \geq 1.05, \mathrm{SBP} \geq 1.06, \mathrm{DBP} \geq$ 1.03 , or NTT $\geq 1.02$. Otherwise, a value of 0 was assigned for that dependent variable.

Statistical significance was accepted at $p<0.05$, except for the tests of HR, SBP, DBP and NTT, which were accepted at $p<0.05 / 4=0.0125$ by Bonferroni correction. SPSS software was used for the statistical analyses.

\section{RESULTS}

\subsection{Characteristics}

The basic characteristics of the subjects as sex, age, and IMAT score did not differ between the FPIL and WPIL groups (Table 1). Environmental conditions such as temperature and humidity were not significantly different between FPIL and WPIL groups (data not shown). The recorded atmospheric pressures on the days of ex- periments were $1015.50 \pm 4.94 \mathrm{hPa}$, and did not differ between subject groups.

\subsection{Anxiety and Autonomic Nervous Activity}

AI score, HR, SBP and DBP significantly increased during/after the MA test compared with the values before the test, whereas NTT significantly decreased after the MA test (Table 2).

Five logistic regression models for predicting the changes in AI score, HR, SBP, DBP and NTT are presented in Table 3. The multivariate-adjusted models showed that the PIL score before the MA test was significant for predicting the change in AI score and the change in HR. The other variables of age, sex, AI score before MA, HR before MA test, SBP before MA test, DBP before MA test, and NTT before MA test were not significant in any of the multivariate-adjusted models.

\section{DISCUSSION}

We found that MA was a possible psychological stressor because AI scores, HR, SBP, DBP and NTT all were changed significantly by the test (Table 2). These results are compatible with previous reports that psychological stresses caused anxiety and also activated the sympathetic nervous system [12]. PIL score was negatively associated with change in AI score and change in HR (Table 3). Therefore, it can be concluded that the persons with a firm PIL showed lower anxiety and a lesser sympathetic nervous response to psychological

Table 1. Demographic and psychological characteristics of subjects by categories of purpose in life (PIL).

\begin{tabular}{|c|c|c|c|}
\hline Characteristic & FPIL group & WPIL group & $p$ \\
\hline $\mathrm{N}$ (male/female) & $26(6 / 20)$ & $33(13 / 20)$ & 0.183 \\
\hline Age (year) & $30.19 \pm 13.02$ & $31.39 \pm 11.50$ & 0.708 \\
\hline PIL test score (point) & $61.12 \pm 4.32$ & $47.24 \pm 7.28$ & - \\
\hline IMAT score (point) & $2.81 \pm 0.98$ & $2.76 \pm 1.35$ & 0.692 \\
\hline
\end{tabular}

N: number of subjects, FPIL: firm PIL, WPIL: weak PIL, IMAT: interest in mental arithmetic test. Data are expressed as mean \pm standard deviation.

Table 2. Anxiety inventory (AI) score, heart rate (HR), systolic blood pressure (SBP), diastolic blood pressure (DBP) and nose tip temperature (NTT) $(\mathrm{N}=59)$.

\begin{tabular}{|c|c|c|c|}
\hline \multirow{2}{*}{ Characteristic } & \multicolumn{3}{|c|}{ Changes } \\
\hline & Before MA & During/After MA & $p$ \\
\hline AI score (point) & $2.05 \pm 1.32$ & $3.20 \pm 1.54$ & $<0.001$ \\
\hline HR (beat/min) & $64.03 \pm 9.86$ & $67.59 \pm 12.14$ & $<0.0025^{\#}$ \\
\hline $\mathrm{SBP}(\mathrm{mmHg})$ & $107.49 \pm 15.87$ & $112.86 \pm 16.73$ & $<0.0025^{\#}$ \\
\hline $\mathrm{DBP}(\mathrm{mmHg})$ & $72.88 \pm 10.29$ & $74.98 \pm 10.47$ & $<0.0125^{\#}$ \\
\hline $\operatorname{NTT}\left({ }^{\circ} \mathrm{C}\right)$ & $33.85 \pm 1.13$ & $33.31 \pm 1.35$ & $<0.0025^{\#}$ \\
\hline
\end{tabular}

MA: mental arithmetic. ${ }^{\#}$ : statistically significant after Bonferroni correction for HR, SPB, DBP and NTT. 
Table 3. Adjusted odds ratios (OR) of demographic, psychological, and physiological characteristics $(\mathrm{N}=59)$.

\begin{tabular}{|c|c|c|c|c|c|c|c|c|c|c|}
\hline \multirow{2}{*}{ Characteristic } & \multicolumn{2}{|c|}{ Change in AI score } & \multicolumn{2}{|c|}{ Change in HR } & \multicolumn{2}{|c|}{ Change in SBP } & \multicolumn{2}{|c|}{ Change in DBP } & \multicolumn{2}{|c|}{ Change in NTT } \\
\hline & OR & $p$ & OR & $p$ & OR & $P$ & OR & $p$ & OR & $p$ \\
\hline Age (year) & 1.04 & 0.155 & 0.97 & 0.238 & 1.04 & 0.135 & 0.99 & 0.790 & 1.03 & 0.205 \\
\hline Sex & 1.38 & 0.619 & 4.62 & 0.046 & 0.51 & 0.392 & 1.03 & 0.968 & 3.21 & 0.079 \\
\hline PIL test score before MA & 0.27 & $<0.05$ & 0.18 & $<0.0125^{\#}$ & 0.76 & 0.662 & 0.96 & 0.939 & 0.33 & 0.076 \\
\hline AI score before MA & 0.51 & 0.296 & 2.61 & 0.150 & 1.99 & 0.275 & 1.52 & 0.477 & 1.51 & 0.493 \\
\hline HR before MA & & & 0.95 & 0.179 & & & & & & \\
\hline SBP before MA & & & & & 0.97 & 0.229 & & & & \\
\hline DBP before MA & & & & & & & 0.94 & 0.057 & & \\
\hline NTT before MA & & & & & & & & & 0.93 & 0.780 \\
\hline
\end{tabular}

\#: statistically significant after Bonferroni correction.

stress. The results are consistent with the previous study [5].

Much attention has been focused on the biological responses caused by psychological stress [13-21]. Sympathetic nervous activity in response to stressors easily generates reactive oxygen species $[1,2,22]$, which acelerate lipid oxidation, gene dysfunction, and telomere shortening [14-17]. It is interesting that the secretions of dopamine and $\beta$-endorphin could be accelerated by the psychological reactions as reward, satisfaction, and/or pleasure based on intrinsic motivation or reinforcement of motivation [18-21]. A lower response against the psychological stresses with FPIL, therefore, could contribute to longevity.

Limitations in this study should be noted. First, each of the AI tests before and during MA and the IMA test consisted of only one question. The tests should be validated for the number of questions, sensitivity, specificity, and reproducibility in the future. Second, since the psychological condition of subjects might influence their test results, the experimental conditions, including instructors' attitudes, should be carefully discussed. Third, food intake before the experiment might influence the autonomic nervous system. Further studies with a large number of volunteers would be required.

In conclusion, we found that a firm PIL decreased anxiety during psychological stress caused by the MA test even under the objective evaluation by others. Having a firm PIL also lowered the response of the sympathetic nervous system to psychological stress.

\section{ACKNOWLEDGEMENTS}

This study was supported by a Grant-in-Aid for Science Research from the Japanese Society for the Promotion of Science (No.17927020; 2005). The authors wish to express their gratitude to Yuko Ishida for the data analysis.

\section{REFERENCES}

[1] Smith, E.E., Nolen-Hoeksema, S., Fredrickson, B.K. and
Loftus, G.R. (2003) Atkinson \& Hilgard's introduction to psychology. Thomson.

[2] Atkinson, R.L., Atkinson, R.C., Smith, E.E., Ben, D.J. and Nolen-Hosksema, S. (1996) Hilgard's introduction to psychology. Harcourt Brace College Publishers, Fort Worth.

[3] Frankl, V.E. (1972) The meaning of meaninglessness: A challenge to psychotherapy. American Journal of Psychoanalysis, 32, 85-89. doi:10.1007/BF01872487

[4] Bundra, A. (1977) Social learning theory. Prentice Hall, Upper Saddle River.

[5] Ishida, R. and Okada, M. (2006) Effects of a firm purpose in life on anxiety and sympathetic nervous activity caused by emotional stress: Assessment by psycho-physiological method. Stress and Health, 22, 275-281. doi:10.1002/smi.1095

[6] Agbu, J.F. (2010) Type A behavior pattern: A new insight to gender challenges in higher education. Psychology, Health \& Medicine, 15, 528-539. doi:10.1080/13548506.2010.487106

[7] Hemingway, H., Shipley, M., Brunner, E., Britton, A., Malik, A. and Marmot, M. (2005) Does autonomic function link social position to coronary risk? Circulation, 111, 3071-3077. doi:10.1161/CIRCULATIONAHA.104.497347

[8] Atanackovic, D., Schulze, J., kröger, H., Brunner-Weinzierl, M.C. and Deter, H.C. (2003) Acute psychological stress induces a prolonged suppression of the production of reactive oxygen species by phagocytes. Journal of Neuroimmunology, 142, 159-165. doi:10.1016/S0165-5728(03)00267-4

[9] Crumbaugh, J.C. and Maholic, L.T. (1964) An experimental study in existentialism: The psychometric approach to Frankl's concept of noogenic neurosis. Journal of Clinical Psychology, 20, 200-207. doi:10.1002/1097-4679(196404)20:2<200::AID-JCLP22 70200203>3.0.CO;2-U

[10] Sato, F. and Tanaka, H. (1974) An experimental study on the existential aspect of life: Part I. Tohoku Psychologica Folia. 33, 20-46.

[11] Ishida, R. (2008a) Correlation between social desirability and autonomic nervous function under goal-oriented stress (mental arithmetic) with consideration of parental attitude (in Japanese). Autonomic Nervous System, 45, 242-249.

[12] Smith, E.E., Nolen-Hoeksema, S., Fredrickson, B.L. and Loftus, G.R. (2003) Atkinson \& Hilgard's introduction to 
psychology. Thomson.

[13] Tannno, K., Sakata, K., Ohsawa, M., Onoda, T., Itai, K., Yaegashi, Y. and Tamakoshi, A. (2009) Association of ikigai as a positive psychological factor with all-cause mortality and cause-specific mortality among middleaged and elderly Japanese people: Finding from the Japan collaborative cohort study. Journal of Psychosomatic Research, 67, 67-75.

doi:10.1016/j.jpsychores.2008.10.018

[14] Willls, L.P. and Schnellmann, R.G. (2011) Telomeres and telomerase in renal health. Journal of the American Society of Nephrology, 22, 39-41. doi:10.1681/ASN.2010060662

[15] Ben-Porath, I. and Weinberger, R.A. (2005) The signals and pathways activating cellular senescence. The International Journal of Biochemistry \& Cell Biology, 37, 961-976. doi:10.1016/j.biocel.2004.10.013

[16] Bartholomew, J.N. and Galbiati, F. (2010) Mapping of oxidative stress response elements of the caveolin-1 promoter. Methods in molecular biology, 594, 409-423. doi:10.1007/978-1-60761-411-1 29

[17] Ishii, N., Ishii, T. and Hartman, P.S. (2007) The role of the electron transport SDHC gene on lifespan and cancer. Mitochondrion, 7, 24-28. doi:10.1016/j.mito.2006.11.012

[18] Stefano, G.B., Bianchi, E., Guarma, M., Fricchione, G.L.,
Zhu, W., Cadet, P., Mantione, K.J., Casares, F.M., Kream, R.M. and Esch, T. (2007) Nicotine, alcohol and cocaine coupling to reward processes via endogenous morphine signaling: The dopamine-morphine. Medical Science Monitor: International Medical Journal of Experimental and Clinical Research, 13, 91-102.

[19] Xu, X., Aron, A., Brown, L., Cao, G., Feng, T. and Weng, X. (2011) Reward and motivation system: A brain mapping study of early-stage intense romantic love in Chinese participants. Human Brain Mapping, 32, 249-257. doi: $10.1002 / \mathrm{hbm} .21017$

[20] Hegadoren, K.M., O’Donnell, T., Lanius, R., Coupland, N.J. and Lacaze-Masmonteil, N. (2009) The role of betaendorphin in the pathophysiology of major depression. Neuropeptides, 43, 341-353. doi:10.1016/j.npep.2009.06.004

[21] Spanagel, R., Herz, A., Bals-Kubik, R. and Shippenberg, T.S. (1991) Beta-endorphin-induced locomotor stimulation and reinforcement are associated with an increase in dopamine release in the nucleus accumbens. Psychopharmacology, 104, 51-56. doi:10.1007/BF02244553

[22] Touyz, R.M. and Briones, A.M. (2011) Reactive oxygen species and vascular biology: Implications in human hypertension. Hypertension Research, 34, 5-14. doi:10.1038/hr.2010.201

Appendix 1. Examples of the questions used in the purpose in life (PIL) test.

(1) In life I have:

$\begin{array}{cccccc}1 & 3 & 4 & 5 & 6 & 7 \\ \text { (no goals or aims at all) } & & \text { (neutral) } & & \text { (very clear goals and aims) }\end{array}$

(2) Concerning man's freedom to make his own choices, I believe man is:

$\begin{array}{cccccc}1 & 3 & 4 & 5 & 6 & 7 \\ \begin{array}{c}\text { (completely bound by limitations } \\ \text { of heredity and environment) }\end{array} & & \text { (neutral) } & \text { (absolutely free to make all choices) }\end{array}$

(3) Every day is

$\begin{array}{lcccccc}1 & 2 & 3 & 4 & 5 & 6 & 7 \\ \text { (exactly the same) } & & \text { (neutral) } & & \text { (constantly new and different) }\end{array}$

(4) My life is:
1
2
3
4
5
6
7
(empty, filled only with despair)
(neutral)
(running over with exciting and good things)

(5) If I should die today, I would feel that my life has been:

$\begin{array}{lrrrrr}1 & 3 & 4 & 5 & 6 & 7 \\ \text { (completely worthless) } & & \text { (neutral) } & & & \text { (very worthwhile) }\end{array}$

